\title{
SINGLE AND COMBINED ABIOTIC STRESSES IMPACT THE ROOT EXUDATION PATTERNS IN DIFFERENT MAIZE ROOT TYPES
}

Raphael Tiziani ${ }^{1}$, Maria Begona Miras Moreno ${ }^{2}$, Antonino Malacrinò ${ }^{3}$, Rosa Vescio ${ }^{4}$, Luigi Lucini $^{5}$, Tanja Mimmo ${ }^{6}$, Stefano Cesco ${ }^{1}$, and AGOSTINO SORGONA ${ }^{4}$

${ }^{1}$ Free University of Bozen-Bolzano

${ }^{2}$ Università Cattolica del Sacro Cuore Facoltà di Scienze Agrarie Alimentari e Ambientali

${ }^{3}$ Westfälische Wilhelms-Universität Münster

${ }^{4}$ Università degli Studi Mediterranea di Reggio Calabria

${ }^{5}$ Università Cattolica del Sacro Cuore

${ }^{6}$ Libera Universita di Bolzano

July 25, 2021

\begin{abstract}
Root exudates play an essential role in plant-soil-abiotic stress interactions. However, we still know little about the influence of stress combinations on the root exudation profile. Using targeted and untargeted metabolomics, here we test the effect of drought, heat stress, and their combination on the maize root exudates, also considering the differences that might exist between root types (seminal and primary) and root zones (apical and sub-apical). In addition, we built an analytical framework that relate the root exudation profile with the characterization of the rhizosphere bacterial community, enabling us to dissect the interactions between specific root exudates and microbial taxa. Our results suggest that the composition of root exudates has a different outcome according to the single or combined stress and to the root zone but not between root types. Further, we found that stress-specific exudates influence the relative abundance of specific microbial taxa, some of which are known to be beneficial microorganisms. Therefore, the stress-specific root exudate composition selecting specific microbial taxa, here observed, represent a contribute on the effects of climate changes on crops increasing thus the potential impact on the current trend of crafting agricultural practices within a wider point of view of plants-microbe-environment interactions.
\end{abstract}

SINGLE AND COMBINED ABIOTIC STRESSES IMPACT THE ROOT EXUDATION PATTERNS IN DIFFERENT MAIZE ROOT TYPES

Raphael Tiziani ${ }^{1,+}$, Begoña Miras-Moreno ${ }^{2,+}$, Antonino Malacrino ${ }^{3}$, Rosa Vescio ${ }^{4}$, Luigi Lucini $^{2}$, Tanja Mimmo ${ }^{1}$, Stefano Cesco ${ }^{1}$, Agostino Sorgona ${ }^{4}$

${ }^{1}$ Faculty of Science and Technology, Free University of Bolzano, 39100 Bolzano, Italy

${ }^{2}$ Department for Sustainable Food Process, Universita Cattolica del Sacro Cuore, 29122 Piacenza, Italy

${ }^{3}$ Institute for Evolution and Biodiversity, Westfalische Wilhelms-Universitat Munster, Munster, Germany

${ }^{4}$ Dipartimento Agraria, Universita Mediterranea di Reggio Calabria, Localita Feo di Vito, 89122 Reggio Calabria 
*Corresponding author: asorgona@unirc.it

+ Equal contributing first-co-authorship

\begin{abstract}
Root exudates play an essential role in plant-soil-abiotic stress interactions. However, we still know little about the influence of stress combinations on the root exudation profile. Using targeted and untargeted metabolomics, here we test the effect of drought, heat stress, and their combination on the maize root exudates, also considering the differences that might exist between root types (seminal and primary) and root zones (apical and sub-apical). In addition, we built an analytical framework that relate the root exudation profile with the characterization of the rhizosphere bacterial community, enabling us to dissect the interactions between specific root exudates and microbial taxa. Our results suggest that the composition of root exudates has a different outcome according to the single or combined stress and to the root zone but not between root types. Further, we found that stress-specific exudates influence the relative abundance of specific microbial taxa, some of which are known to be beneficial microorganisms. Therefore, the stressspecific root exudate composition selecting specific microbial taxa, here observed, represent a contribute on the effects of climate changes on crops increasing thus the potential impact on the current trend of crafting agricultural practices within a wider point of view of plants-microbe-environment interactions.
\end{abstract}

Keywords: root exudation profile, heat, drought, abiotic stress, microbiota, metabolomics,

\title{
INTRODUCTION
}

The reduction of water availability (i.e ., drought stress) and increase in global temperature (i.e . heat stress) are among the major challenges we are going to face in the next few decades (IPCC 2018). These abiotic stresses generated by climate change significantly impact plants (Pandey, Ramegowda \& Senthil-Kumar 2015), causing substantial agronomical and economic losses every year on a global scale (Lipiec, Doussan, Nosalewicz \& Kondracka 2013). The effect of abiotic stresses on plants has been widely dissected over the years. However, plants are rarely exposed to single stresses but rather to a combination of multiple stresses, requiring plants to finely tune their physiological responses. Several studies have shown that combined stress responses provide non-additive effects (i.e., synergistic or antagonistic), and therefore the combined effects cannot be predicted based on results from single-stress studies (Rivero et al. 2014). Hence, it is of huge importance to understand how plants respond to the combination of heat and drought stress.

One of the most important physiological traits that plants evolved to interact with the environment is the modulation of their root exudation pattern (Vives-Peris, de Ollas, Gomez-Cadenas \& Perez-Clemente 2019). Root exudates include primary and secondary metabolites of both low $(<1000 \mathrm{Da})$ and high molecular weight (>1000 Da) (Oburger \& Jones 2018) that plants produce to directly influence the chemical, physical and biological characteristics of the rhizosphere (Mommer, Hinsinger, Prigent-Combaret \& Visser 2016). Examples include carboxylates, amino acids, carbohydrates, phenols, proteins, and fatty acids. Plant age, genotype, biotic or abiotic stresses, environmental factors, as well as the interaction with living organisms in the rhizosphere can potentially affect the quantity and quality of root exudation (Czarnota, Rimando \& Weston 2003; Pii et al. 2015; Valentinuzzi et al. 2015; Oburger \& Jones 2018; Tiziani et al. 2020a; Tiziani, Pii, Celletti, Cesco \& Mimmo 2020b). Despite the large interest in drought- and heat-tolerant crops in the last years, only a few studies focused on the root exudation responses to these abiotic stresses (Williams \& de Vries 2020).

In the presence of water deficit it has been suggested that plants allocate a higher fraction of carbon to exudates at the expenses of biomass production, although it seems that total exuded carbon is limited during the drought stress (Karst, Gaster, Wiley \& Landhausser 2017; Preece, Farre-Armengol, Llusia \& Penuelas 2018). A recent study investigated drought effects on root exudate quality and quantity in sunflower and soybean. Sunflower plants exposed to drought increased the exudation rates after rewetting $(+330 \%$ in terms of carbon), but the composition of metabolites remained unchanged compared to not-stressed plants. In soybean, it has been observed that plants did not modify exudation rates, but rather the profile of 
metabolites exuded, with increased concentrations of osmolytes (proline and pinitol) (Canarini, Merchant \& Dijkstra 2016). Another study performed on Quercus ilex showed that under normal growing conditions, the plant mainly exuded primary metabolites, whereas under drought stress, the exudation was shifted irreversibly to secondary metabolites (Gargallo-Garrigaet al. 2018). In contrast, Henry, Doucette, Norton \& Bugbee (2007) showed that drought stress in wheatgrass increased carboxylate exudation, especially malate, compared to control plants. Taken together, the plant responses to drought often seem to be species- or even genotype-specific. Research on the effects of heat stress on root exudation is extremely scarce, while effects like decreased root growth and function, including nutrient and water uptake, have been widely described (Heckathorn, Giri, Mishra \& Bista 2013). Under salt or heat stress, citrus plants have been shown to release proline in larger quantities from roots during heat stress (Vives-Peris, Molina, Segura, Gomez-Cadenas \& Perez-Clemente 2018). Furthermore, the exudation of the phytohormones salicylic acid, indole acetic acid, abscisic acid, and jasmonic acid generally increased under elevated temperatures (Vives-Peris et al. 2018a). Another recent study examined the effect of heat on Sorghum bicolor root exudation, highlighting that heatstressed plants exuded large amounts of compounds, including ascorbate, carotene, glutathione, or flavonoids (Yaqoob, Bhatti, Sultana \& Shahid 2020). Considering that the effects of the single stress, as water deficit and high temperatures, on the root exudation have been reported, we still know little about this essential physiological process to the combination of these abiotic stress despite that they co-occur in nature and determine a unique plant response that is different from each single stress.

Most studies regarding the root exudation process have been focused on exudate profiles of the whole root system. However, the plant root system comprises different root classes or types, such as embryonic (primary and seminal) and post-embryonic roots (nodal and lateral), which are characterized by distinct developmental, physiological, and functional signatures (Bengough 2003). For example, several studies reported different morpho-physiological responses among root types to drought stress (Zhan, Schneider \& Lynch 2015), allelochemicals (Abenavoli, Sorgona, Albano \& Cacco 2004; Lupini, Sorgona, Princi, Sunseri \& Abenavoli 2016), P deficiency (Rubio, Sorgona \& Lynch 2004; Tiziani et al. 2020a) and combined nitrogen deficiency/drought stress (Lynch 2013). In addition to this diversification between root classes/types, roots also show differences in functionality along their axis (Rubio et al. 2004; Sorgona et al. 2011; Tizianiet al. 2020a). Despite this functional complexity of the root system, very few studies considered the within-root variability of the exudation processes. Specifically, the carbon efflux is generally highest at the root tips (McCully and Canny, 1985; Iijima et al., 2000). However, it has been observed that branch roots released more flavonoids in white clover (Mathesius et al., 2000) and tryptophan in annual grass, although the apical region of the primary root emitted more sucrose (Jaeger et al., 1999). Understanding the variation of exudate profiles among and along the diverse root types will allow predicting the impact of the root architecture on biophysical and edaphic properties (aggregation, structure, $\mathrm{pH}$, moisture, temperature, and nutrient stoichiometry) in different soil locations.

In addition to the effects on the physio-chemical proprieties of the rhizosphere, root exudation is a key process through which plants interact with soil microbes and, consequently, influencing several other rhizosphere processes. Despite the recent increase of research on root exudation and their link with rhizosphere microbial communities (de Vries et al. 2018; Gargallo-Garriga et al. 2018; Karlowskyet al. 2018; Vescio et al., 2021), until now the relationship between exudation pattern and microbial responses under drought and heat stress, in single and combination, has been largely unexplored.

In this framework, in the present study we test the effect of drought, heat stress, and their combination on the root exudation profile of maize plants, also considering the differences that might exist between root types (seminal and primary) and root zones (apical and sub-apical). According to previous studies, we hypothesize that (i) drought and heat stress will induce a differential response in the blend of root exudates; (ii) the combination of the two stresses will generate a different exudation profile than each single stress; (iii) diverse root types and zones will point out a different exudate pattern for each single or combine stress. Furthermore, to provide a mechanistic overview of the role of root exudates in rhizosphere processes, here we leverage data generated by our previous study (Vescio et al. 2021). In particular, we aim at unraveling the interaction between root exudates and the rhizosphere bacterial microbiota when maize plants are exposed to abiotic 
stress. According to previous studies suggesting a role of root exudates in recruiting beneficial microbes, we hypothesize that (iv) stress-specific root exudates will influence the relative abundance of microbial taxa that have a beneficial effect on maize plants.

\section{MATERIAL AND METHODS}

A randomized experimental design was used throughout the entire experiment, to include a total of 4 abiotic stress treatments ( 2 water availability treatments $\mathrm{x} 2$ air temperature treatments) x 2 root types (primary and seminal roots) $\mathrm{x} 2$ root zones (apical and subapical) $\mathrm{x} 5$ (replicates) $=80$ samples.

\section{Plant growth and exudates sampling}

Samples collected for this experiment were part of our previous study (Vescio et al., 2021) conducted using maize plants (Zea mays L., genotype KXB7554 provided by KWS Italia) as a model. Plants were grown in pots filled with a mix of collected field soil and quartz sand (1:2, v:v). Quartz sand (O1-2 mm, Croci Trading Company s.r.l., Italy) was previously autoclaved for 3 hours at $121 \mathrm{degC}$, allowed to cool overnight, and then autoclaved further 3 hours at $121 \mathrm{degC}$. The soil physicochemical parameters are reported in Gelsomino et al. (2010). Plants were initially grown for 2 weeks at $25 \mathrm{degC}, 70 \%$ relative humidity and at $14 \mathrm{~h}: 10 \mathrm{~h}$ light:dark photoperiod. Then, plants were exposed to two levels of water availability ( $30 \%$ and $80 \%$ of soil field capacity, corresponding to a severe drought and no drought) and air temperature (25degC and $32 \mathrm{degC}$ ) for one week. Finally, the following abiotic stress treatments were obtained: $25 \mathrm{degC}$ and $80 \%$ soil field capacity (T1 or control); $25 \mathrm{degC}$ and $30 \%$ soil field capacity (T2 or drought stress); 32degC and $80 \%$ soil field capacity (T3 or heat stress); $32 \mathrm{degC}$ and $30 \%$ soil field capacity (T4 or combined stress).

\section{Root exudates sampling}

After 7 days of exposure to treatments, root exudates were collected by a soil-hydroponic hybrid approach (Oburger \& Jones 2018) with modifications (Figure 1). The maize plants were sampled and the whole root system was carefully washed after removing the soil. Afterwards, the single primary and seminal roots attached to the shoot were placed on two different compartmented chambers $(2 \times 10 \times 2 \mathrm{~cm})$ with five cells, each $1.8 \mathrm{~cm}$ deep and $1.8 \mathrm{~cm}$ wide (Figure 1). Each cell was connected to the next one by a shallow bridge so that a root could be laid out across the block traversing all the cells. The intact primary and seminal root axes were inserted in separate chambers. The area starting from the root tip to the first visible lateral root (apical root zone) was isolated from the remaining root portion (subapical root zone) by silicon grease (Dow Corning, Midland, MI, USA) (Figure 1). Hence, the root exudates of the following root types and zones have been collected: seminal-apical (Sa), seminal-subapical (Ssa), primary-apical (Pa), and primary-subapical (Psa).

Before beginning the exudate collection, we submerged the apical root zone with the trap solution $(0.5 \mathrm{mM}$ $\mathrm{CaCl}_{2}$ ) to detect possible leaks between the cells hosting the apical and sub-apical root zones. During the exudates collection, the roots were continuously aerated. To collect root exudates, cells were filled with 6 $\mathrm{mL}$ of the trap solution, and every 30 minutes the trap solution was collected for up to 4 hours, yielding $48 \mathrm{~mL}$ for each root zone. This solution was then centrifuged (15 min at $13000 \mathrm{rpm}$ at $4 \mathrm{degC}$ ) and the supernatant was freeze-dried. The exudate solution was then resuspended in $5 \mathrm{~mL} 1: 1 \mathrm{H}_{2} \mathrm{O} / \mathrm{CH}_{3} \mathrm{OH}$ and filtered with $0.45 \mu \mathrm{m}$ syringe filters (Phenex-RC $0.45 \mu \mathrm{m}$ - Phenomenex) and used for the targeted and untargeted metabolomics analyses.

\section{Targeted exudates analysis}

\section{Determination of total phenols}

Total phenol content was determined following the Folin-Ciocalteau method (Atanassova, Georgieva \& Ivancheva 2011). Briefly, $200 \mu \mathrm{L}$ of sample or standard solution of gallic acid was mixed with $1.4 \mathrm{~mL}$ deionized water and $100 \mu \mathrm{L}$ Folin-Ciocalteau's phenol reagent (2 mol L ${ }^{-1}$; Sigma Aldrich; Italy). Total phenol content was determined using a spectrophotometer (Agilent Technologies, Cary Series 100 UV-VIS Spectrophotometer, Italy) to measure the absorbance at $765 \mathrm{~nm}$, then expressing the results as $\mu \mathrm{mol}$ of gallic acid equivalent. 


\section{Determination of total flavonoids}

Total flavonoids content was determined by a pharmacopeia method (Miliauskas, Venskutonis \& van Beek 2004). Rutin hydrate (purity [?] 94\%, Sigma Aldrich; Italy) was used as reference compound. Briefly, 160 $\mu \mathrm{L}$ of sample or a standard solution of rutin was mixed with $160 \mu \mathrm{L} \mathrm{AlCl} 3\left(20 \mathrm{~g} \mathrm{~L}^{-1}\right.$ in ethanol; Sigma Aldrich; Italy) and $3.68 \mathrm{~mL}$ ethanol (Sigma Aldrich; Italy). Samples and standards were incubated at $20{ }^{\circ} \mathrm{C}$ for $40 \mathrm{~min}$, and absorbance read at $415 \mathrm{~nm}$ using a spectrophotometer (Agilent Technologies, Cary Series 100 UV-VIS Spectrophotometer, Italy). Results were expressed $\mu$ mol rutin equivalent.

\section{Determination of total flavonols}

The content of total flavonols was determined following the Yermakov method (Mickelsen \& Yamamoto 1958). Rutin hydrate (Sigma Aldrich, purity [?] 94\%) was used as standard solution. Briefly, $200 \mu \mathrm{L}$ of sample or rutin standard was mixed with $200 \mu \mathrm{L} \mathrm{AlCl3}\left(20 \mathrm{~g} \mathrm{~L}^{-1}\right.$ in ethanol, Sigma Aldrich) and $600 \mu \mathrm{L}$ sodium acetate $\left(50 \mathrm{~g} \mathrm{~L}^{-1}\right.$; Sigma Aldrich). Samples and standards were incubated at $20{ }^{\circ} \mathrm{C}$ for $2.5 \mathrm{~h}$, and absorbance measured at $440 \mathrm{~nm}$ using a spectrophotometer (Agilent Technologies, Cary Series 100 UV-VIS Spectrophotometer, Italy). Results were expressed as $\mu \mathrm{mol}$ rutin equivalent.

\section{Determination of organic acids}

Organic acids were determined by ionic chromatography with photodiode array detection (Waters 2998, Waters Corporation, Italy). Separation was achieved on a cation exchange column (Aminex 87-H column, $300 \times 7.8 \mathrm{~mm}, 9 \mu \mathrm{m}$, Bio-Rad) using an isocratic elution with $10 \mathrm{mM} \mathrm{H}_{2} \mathrm{SO}_{4}$ as carrier, at a flow rate of $0.6 \mathrm{~mL} \mathrm{~min}{ }^{-1}$. Detection was carried out at $210 \mathrm{~nm}$. Standard carboxylates were prepared as individual stock solutions using Sigma (Italy) reagent grade compounds, and then combined to give diluted working standards to be used for retention time comparison and for quantification.

\section{Determination of amino acids}

Amino acids analysis was performed by liquid chromatography prior a pre-column derivatization with a commercial kit (AccQ Tag, Waters Corporation, Italy) according to manufacturer's instructions and using a Nova-PakTMC18 silica based bonded column (Waters Corporation, Italy). Gradient elution with a flow rate of $1.0 \mathrm{~mL} \mathrm{~min}{ }^{-1}$ used a mixture of: $\mathrm{A}=\mathrm{AccQ}$ Tag Eluent; $\mathrm{B}=100 \%$ acetonitrile (HPLC grade), $\mathrm{C}$ $=$ HPLC grade I water (Waters application note for amino acids). Gradient summary: 100:0 A/B to 83:17 $\mathrm{A} / \mathrm{B}$ from min 0 to 38 . Then $60: 40 \mathrm{~B} / \mathrm{C}$ from min 38.01 to 55 . The derivatized amino acids were detected by fluorescence $\left(\lambda_{\text {ex }}=250 \mathrm{~nm}, \lambda_{\text {ems }}=395 \mathrm{~nm} ; 2475\right.$ Multi $\lambda$ Fluorescence Detector, Waters Corporation, Italy) and quantified by the external standard method using a pure amino acids standard mix.

\section{Untargeted exudate analysis}

Rood exudate composition was investigated trough an ultra-high-pressure liquid chromatography (UHPLC) coupled to a quadrupole-time-of-flight (QTOF) mass spectrometry as reported by Astolfi et al. (2020) with minor modifications. Briefly, a 1290 series LC system equipped with a binary pump, a JetStream Electrospray source and a G6550 iFunnel QTOF mass spectrometer (Agilent technologies, Santa Clara, CA, USA) working in positive SCAN mode (100-1200 m/z range) was used. The chromatographic separation was achieved by a reverse phase Agilent PFP column $(2.0 \times 100 \mathrm{~mm}, 3 \mu \mathrm{m})$ using a gradient of water and methanol (from $6 \%$ to $94 \%$ organic phase in $33 \mathrm{~min}$, flow rate $200 \mu \mathrm{lmin}{ }^{-1}$ ). Quality control pooled samples (QCs) were also analyzed in MS/MS mode (data-dependent, 12 precursors per cycle at $1 \mathrm{~Hz}, 50-1000 \mathrm{~m} / \mathrm{z}$, positive polarity, active exclusion after 2 spectra), with collision energies of 10,20 , and $40 \mathrm{eV}$ as previously reported (García-Pérez, Miras-Moreno, Lucini \& Gallego 2021).

Compound annotation was carried out according to Astolfi et al.(2020) from raw mass features, using the software Profinder B.07 (from Agilent Technologies, Santa Clara, CA, USA) according to the 'find-byformula' algorithm following mass and retention time alignment. To this aim, the whole isotope pattern (monoisotopic mass, isotopic spacing and isotopic ratio) was used. A custom database produced by combining compounds exported from PlantCyc 9.6 (Plant Metabolic Network, http://www.plantcyc.org; accessed April 
2017), Phenol-Explorer 3.6 (http://www.phenol-explorer.eu; accessed April 2017), as well as information on compounds extracted from the literature and that might be present in root exudates was used as a reference for annotation (5-ppm mass accuracy tolerance). The annotation level corresponded to Level 2 of accuracy (putatively annotated compounds) as set out by the COSMOS Metabolomics Standards Initiative (http://cosmos-fp7.eu/msi). Only those compounds identified within $75 \%$ of replications within at least one treatment were retained.

The MS-DIAL 4.24 software provided a higher degree of confidence in annotation, based on MS/MS spectra of QCs to confirm features obtained by Profinder B.07. (Tsugawa et al. 2015). To this aim, publicly available built-in MS/MS experimental spectra (Mass Bank of North America) were used.

\section{Statistical analysis}

All data from targeted analyses were tested for normality (Kolmogorov-Smirnov test) and for homogeneity of variance (Leven median test). Thereafter, three-way ANOVA was used to investigate the effect of treatments (control, drought, heat, and drought+heat), root type (primary and seminal), root zones (apical and subapical) and their interactions. The means were separated by the Tukey's honest significant difference (HSD) test $(\mathrm{p}<0.05)$.

The metabolomics-based analyses were performed according to García Pérezet al. (2021). Briefly, raw mass features were elaborated using the software Agilent Mass Profiler Professional B.12.06 for normalization and baselining, and then the multivariate unsupervised hierarchical cluster analysis (HCA) was performed (Euclidean distance, Ward's linkage rule) to describe similarities and dissimilarities among treatments from a fold-change based heat map. A Volcano analysis $(\alpha=0.05$; fold change, FC $>1.2)$ was performed to identify compounds varying significantly between treatments and control. In addition, supervised modeling by Orthogonal Projection to Latent Structure Discriminant Analysis (OPLS-DA) was performed using SIMCA 16 software tool (Umetrics, Sweden). The Variable Importance in Projection (VIP) analysis was performed to further identify markers responsible of the discrimination. The obtained multivariate model was then cross-validated by Cross Validation-Analysis of Variance (CV-ANOVA, $\alpha<0.01)$ and its fitness and prediction were evaluated by R2Y and Q2Y parameters, respectively.

\section{Integration of metabolome and microbiota datasets}

The rhizosphere microbiota dataset was taken from our previous experiment (Vescio et al., 2021), where additional maize plants from the present experiment (and the same pot) were used. In the previous work, libraries targeting the V4 region of the bacterial 16S rRNA were obtained from DNA extracted from rhizosphere soil (McPherson et al., 2018), and sequenced using an Illumina MiSeq platform (Illumina, San Diego, CA, USA) using the 300PE chemistry. De-multiplexed forward and reverse reads were merged using the PEAR 0.9.1 algorithm using default parameters (Zhang et al. 2014). Data handling was carried out using VSEARCH 2.14.2 (Rognes et al. 2016) to quality-filtered reads, discard chimeric sequences, bin OTUs and assign taxonomy by querying the SILVA database (v. 132) (Quast et al. 2012).

Data analysis was performed using R statistical software 3.5 (R Core Team 2013) (Supplementary material 1). The microbiota dataset was processed to remove singletons and OTUs generated from the amplification of plastidial rRNA. This dataset was then normalized using DESeq2 (Love, Huber \& Anders 2014). The metabolome dataset was also processed to remove those metabolites that were not found in at least $25 \%$ of the samples. Then, a framework that considers both metabolomic and metabarcoding datasets was built to finely investigate the interaction between root exudates and rhizosphere microbial community. This framework has been built using MOFA+ (Argelaguet et al. 2018). MOFA+ captured three factors that explain a high proportion of variation in both molecules and taxa in the three different treatments (Supplementary material 1). This information allowed identifying, for each treatment, the top $10 \%$ molecules and $0.1 \%$ microbial taxa that mostly contribute to explain the variation due to the treatments. Then, their relative abundance was correlated (Two tails, Pearson's correlation) and significant correlations $(\mathrm{p}<0.05)$ were recorded.

\section{RESULTS}




\section{Targeted exudates analysis}

Figure 2 shows the compounds detected by the targeted metabolomics analysis. We were able to quantify aconitic acid, total phenols, flavonoids and flavonols, as well as glycine, threonine and alanine in all the samples (Figure 2). Formic and lactic acid were also detected, yet only in some random samples. Furthermore, we were able to detect, but not quantify serine, histidine, proline, tyrosine and phenylalanine.

Aconitic acid exudation rate was significantly affected by the treatments (Figure 2a). Plants exposed to T3 exuded 107\% more aconitic acid than T2 plants, while T1 and T4 treatments exhibited intermediate values. Also, the root zones pointed out statistically significant difference of aconitic acid exudation: the apical zone exuded $151 \%$ more aconitic acid than the sub-apical one.

The total phenols (Figure 2b) were affected in the same way by the treatments and root zones: T3 vs T2 $(+120 \%)$, and the other two treatments displayed intermediate exudation rates while the apical zone exuded more $(+157 \%)$ than the sub-apical one (Figure $2 b)$.

On the other hand, the release of total flavonoids was higher in T1 plants (Figure 2c), being nearly 4-fold higher when compared to T2. T3 showed intermediate values and in T4 no flavonoids were detected (<LOD). However, similar to the phenols and aconitic acid exudation, the apical zones exuded more total flavonoids than the sub-apical one $(+295 \%)$.

Threonine exudation rate showed the same trend as the total flavonoids (Figure 2d). Indeed, T1 exuded significantly more threonine compared to T2 (+105\%), while both T3 and T4 exhibited intermediate threonine exudation rates. Once again, the apical zone exudes more threonine than the sub-apical one $(+246 \%$, Figure 2d). Glycine and alanine exudation were significantly affected by root zone being the apical zone more responsive $(+213 \%$ and $+193 \%$, respectively, respect the sub-apical zone) (Figure 2e and f).

Total flavonols exudation rate was not altered by any factor (Figure $2 \mathrm{~g}$ ).

\section{Untargeted metabolomics}

The comprehensive exudate profiling of the different maize root types and zones exposed to single and combined abiotic stress was investigated using UHPLC-QTOF-MS untargeted metabolomic analysis. This approach allowed the putative annotation of 337 compounds, most of which belonged to phenylpropanoids and amino acids (Supplementary material 2). We solely found slight differences driven by root type or root zone (Supplemental material 3. However, the naïve HCA analysis showed a separation of exuded metabolic profiles according to the stress treatments (Figure 3). Therefore, we focused our analysis on understanding the differences among treatments. This latter HCA unsupervised model depicted two main clusters, separating control and drought-stressed plants from plants exposed to heat and combined stress, suggesting that temperature had a hierarchically stronger effect on exudate composition than drought. The supervised OPLS-DA further confirmed this separation as a function of the stress applied (Figure 4). The first latent vector allowed separating control plants and those exposed to drought from heat- and combinedstress plants. However, despite the closeness of exudates profile between control and drought-stressed plants, the second latent vector indicates that also the soil water content influenced exudate signatures (Figure 4). The VIP analysis highlighted the most discriminant compounds involved in OPLS-DA score plot separation (Supplementary material 4). The features having a VIP score $>1$ were considered as markers. Among these compounds, phenylpropanoids appeared as the main class of compounds, possessing the highest VIP score (Supplementary material 4). Nevertheless, several organic acids and amino acids were also highlighted as VIP markers.

To further investigate the exudation pattern as a function of the stress, the volcano analysis was performed, to identify differential metabolites elicited under stress, compared to non-stress conditions. The differential metabolites for each treatment are provided in Supplementary material 5. According to the multivariate statistics (Figure 3 and Figure 4), plants exposed to heat and combined stresses presented the largest differences compared to control plants, pointing out 33 and 39 metabolites, respectively (supplementary material 5). 
Venn analysis (Figure 5) shows that abiotic stress conditions shaped the exudation of compounds compared to the control conditions. Although several compounds are down-exuded in the presence of the stresses, the stress-treated plants presented a more pronounced up-exudation of compounds, especially under combined stress. Indeed, in front of 18 down-exuded metabolites, a total of 37 compounds were up-exuded, with 13 being observed in combined stress (Figure 5). In detail, all the stressed plants showed that among the shared compounds, hispidulin was strongly repressed, while 4-hydroxycoumarin, citramalic acid and malonic acid strongly increased (supplementary material 5).

Table 1 reports the specific and shared down- and up-exuded compounds in presence of single and combined stress. Drought specifically modulated the exudation of phenolic compounds. In particular, the water shortage repressed the exudation of the phenolic acid protocatechuic acid and dihydroisocoumarin mullein, but promoted the exudation of two conjugated flavonoids and the phytoalexin resveratrol (Table 1). The conjugated flavonoid kaempferol 3- $O$-(6"-acetyl-galactoside) $7-O$-rhamnoside was the only metabolite shared with the combined stress (Table 1). Similarly, heat stress largely promoted the exudation of phenolic compounds. In more detail, plants exposed to heat stress reduced the exudation of L-ascorbate, bisdemethoxycurcumin and apigenin 7-O -(6"-malonyl-apiosyl-glucoside) but increased those of 4-vinylphenol, luteolin 7-O -(2apiosyl-6-malonyl)-glucoside, malate, 2,4-dihydroxybenzoic acid and benzoic acid (Table 1). Differently to the drought stress, the heat shared more metabolites with the combined stress: 4 and 8 down- and up-exuded, respectively (table 1 ).

Combined stress triggered a distinct exudation pattern, compared to the control or individual stresses. In this regard, 13 metabolites were up-exuded solely in the presence of the combined stress, most of which belonged to phenolic compounds. Among these, several flavonoids, the hydroxycinnamic acid trisinapoylgentiobiose, alk(en)ylresorcinol and nonadecenylresorcinol were identified. Moreover, palmitic acid, tryptophan, sinapine and the xanthophyll lutein could be highlighted under combined stress conditions (Table 1). A reduced exudation of alpha-aminoadipic acid, sorgolactone and 6-geranylnaringenin was also specifically observed in combined stress (Table 1).

\section{Integration of metabolome and microbiota datasets}

To test our hypothesis in which stress-specific root exudates can influence the relative abundance of microbial taxa, we built a framework that integrates microbiota and metabolome data. This investigation targets at elucidating the influence of each treatment on both exudates and microbiota, and to identify microbial taxa and compounds that may coordinately interact in response to stress(es). Excitingly, the variance decomposition step revealed three factors explaining a high proportion of the variance, jointly for metabolome and microbiota in the three different treatments (Supplementary material 1). For each treatment, we then selected the top $0.1 \%$ of bacterial taxa and the top $10 \%$ of metabolites that mostly contribute to explain the variation within each factor, and then we correlated the abundance of the bacterial taxa and metabolites, then extracting significative correlations.

The analysis of drought-stresses plants suggests that two compounds (p-coumaric acid ethyl ester and Lserine) negatively correlate with several bacterial taxa. Both compounds were negatively correlated with the same pool of taxa (Mucilaginibacter , Sphingomonas ,Acidibacter, Paenibacillus, Filimonas, Labrys, Mesorhizobium, Heliimonas, Rudaea) with the only difference that L-serine negatively correlated with a single unidentified bacterium, while p-coumaric acid ethyl ester negatively correlated with 2 unidentified bacterial OTUs (Supplementary material 1).

Plants exposed to heat treatment showed five compounds [aminocyclopropane-1-carboxilic, rhamnetin, L-histidine, Quercetin 3-O -(6-acetyl-galactoside) 7- $O$-rhamnoside and 2-methoxy-5-prop-1enylphenol/eugenol] correlating negatively with a total of 23 bacterial taxa (Supplementary material 2). Interestingly, the first four molecules negatively correlated with a common pool of 22 taxa (Supplementary Material 1) including the genera Bacillus ,Bacteriovorax, Caulobacter, Chitinophaga ,Dyadobacter, Heliimonas, Mesorhizobium, Micromonospora, Niastella, Pseudonocardia, Rhizobacter together with 6 OTUs of unidentified bacteria. 2-Methoxy-5-prop-1-enylphenol/Eugenol negatively correlated with 4 OTUs: one 
belonging to the genus Alsobacter and three toCaulobacter (Supplementary material 1).

When plants were exposed to combined stress, the analysis suggests that two compounds (L-homocysteine and cinnamoyl glucose) negatively correlate with bacterial taxa, while a positive correlation was observed only for carnosic acid. L-homocysteine influenced the abundance of 25 taxa (Supplementary material 1) including Bacillus ,Clavibacter, Devosia , Dietzia ,Microbacterium, Paenibacillus , Paracoccus ,Rhizobium , Rhodococcus, Stenotrophomonas . Cinnamoyl glucose and carnosic acid affected, in opposite direction, a single OTU of Paenibacillus (Supplementary material 1).

\section{DISCUSSION}

\section{Drought, heat, or combined stress differently affect the root exudate composition}

In field conditions, plants are regularly subjected to co-occurring abiotic stress factors, especially due to climate change (Zandalinas, Mittler, Balfagón, Arbona \& Gómez-Cadenas 2018). In such adverse growing conditions root exudates are crucial in allowing plants to survive (Mimmo et al. 2018; Vives-Peris et al. 2019). Here we show for the first time distinctive responses of maize root exudation to drought and heat stress, as well as to their combination.

Our results indicate that abiotic stresses modified the root exudates composition, in a more pronounced manner for heat and combined stress, but also evident for drought-stressed plants (second latent vector in figure 4). With this regard, phenylpropanoids and amino acids were the main metabolites involved in the stress-changed root exudation profiles. In this sense, literature indicates that root exudates could be modulated by external factors such as abiotic stress, confirming our results. Indeed, it is known that drought affects the quantity and quality of root exudation, with a prevalence of secondary metabolites (Williams and de Vries, 2020; Gargallo-Garriga et al. 2018). Similarly, several authors also reported a tailored exudation when plants are exposed to high temperature (Pramanik et al., 2000; Vives-Peris et al., 2020).

Our results corroborate previous findings and indicate that both shared and specific metabolites were modulated between the single and combined stress. Among the shared metabolites, citramalic, malonic acid, and 4-hydroxycoumarin increased in presence of all stress treatments, compared to the control. Citramalic acid seems to be involved in soil phosphorus solubilization (Khorassani et al. 2011). Since both drought and heat decrease the water content in the rhizosphere, mobility of nutrients (including phosphorus) may be reduced. Thus, the increase of citramalic acid exudation could be a plant mechanism to enhance the solubilization of the drought-induced mobility-limited nutrients, and consequently their uptake. (Zhang, Chen, Zhao, Zhou \& Zhao 2017) observed that citramalic acid was sharply reduced in the root collar tissue of Caragana korshinskii during drought stress, a highly drought tolerant plant, but not in the root system. This result could indicate the involvement of citramalic acid exudation in drought-adaptation in Caragana korshinskii, although further confirmation is needed. Nonetheless, citramalic acid was found to be accumulated in leaves of soybean seedling under salt stress (Zhang, Yang, Li \& Shi 2016), further indicating that it might play a role in plant response to abiotic stresses that involve osmotic imbalance. Another organic acid that was found in the root exudome of all stressed maize plants is malonic acid. Indeed, the malonic acid content increased in exudates derived from stressed plants such as wheatgrass (Henry A., Doucette W., Norton J. \& Bugbee B., 2007) and has been associated with osmotic adjustment and stress response (Li \& Copeland 2000). Moreover, malonic acid is a major competitive inhibitor of succinate dehydrogenase ( $\mathrm{Li}$ \& Copeland 2000) and thus is involved in plant stress signalingvia mitochondrial succinate dehydrogenase (Belt et al.2017). However, Festuca arundinacea as well as seven Triticeae species decreased their malonic acid leave content under heat stress (Yu, Du, Xu \& Huang; Ullah, Yüce, Neslihan Öztürk Gökçe \& Budak 2017). Finally, 4-hydroxycoumarin was another up-exuded metabolite observed in all stressed maize plants. This compound belongs to the coumarins, known also as allelopathic compounds which interact with the root microbiota for improving nutrient acquisition (Harbort et al., 2020) and, in general, for the assembly of the root microbiome (Stringlis et al., 2019).

Differently to the up-exuded compounds, the amino acids L-serine and threonine and flavones, (especially hispidulin) were the down-exuded metabolites being shared in all stress treatments (Supplementary material 
5 and Table 1). In particular, L-serine was clearly down-exuded under both heat and combined stress, suggesting a role of temperature in its production. The release of threonine instead was repressed by both drought and combined stress. The reduction of the amino acid exudation like serine and threonine could be a plant mechanism to preserve important elements, e.g. C and N, within the plant in presence of abiotic stress conditions. Further, plants might compete with microorganisms for carbon within the rhizosphere since they are also able to reacquire root exudates (Tiziani et al 2020). Thus, plants might activate a so-called 'energy saving strategy' modifying their exudation pattern reducing the microbial competition (Doornbos, van Loon \& Bakker 2012). This may indicate that plants provide a fine tuning of the exudation process under heat and drought stress, even more than under normal growing conditions. Hispidulin was among the metabolite suppressed in all stress treatments respect than control (Supplementary 5 and Table 1). Hispidulin is a flavone with antioxidant activity and belongs to the flavonoids commonly present in root exudates (Lucini et al. 2019). Usually, under heat and/or drought stress, shoots increase the production of antioxidant compounds to counteract the oxidative imbalance generated by increased production of reactive oxygen species (Fahad et al. 2017). The strong repression of hispidulin together with the modulation of other flavonoids might indicate that the maize plant focuses on protecting the shoots rather than prioritizing metabolites for root exudation.

Interestingly, specific stress-related exudation responses could be also observed. Targeted metabolomics indicated that the release of aconitic acid and total phenols was significantly higher in heat-treated plants (Figure 2 ), and a specific phenolic, namely 4 -vinylphenol, was observed to increase by untargeted metabolomics (Table 1). Among phenolics, benzoic acid and its derivatives (i.e. 3,5-dihydroxybenzoic acid, 2,4-dihydroxybenzoic acid, benzoic acid) were specifically exuded under heat stress conditions (Table 1). In agreement with our results, benzoic acid and hydroxybenzoic acid were modulated as a response to alteration in temperature in root exudates (Pramanik et al., 2000). It is also known that benzoic acid released by root exudates plays a role in plant-microorganisms interaction (Liu et al., 2015). Malate, another up-exuded compound observed in maize root exudates, has been demonstrated to recruit beneficial microbes like Bacillus subtilis (Thimmaraju et al., 2008). Indeed, this specific bacterium is involved in the enhancement of plant stress tolerance specifically in dry and salty environments (Bokhari et al., 2019). On the other hand, L-ascorbate was specifically down-exuded in heat-treated maize exudates (supplementary material 5 and Table 1). In planta, ascorbic acid is a well-known key antioxidant and signaling molecule involved in mitigating excessive activities of the reactive oxygen species determined by several abiotic stresses (Venkatesh and Park, 2014). It can be postulated that heat-stressed maize plants preserve the presence of this essential compounds within the heat-induced stressed root cells, rather than exude it into rhizosphere.

Among the specific compounds up-exuded by maize roots subjected to drought stress, we observed resveratrol. This compound belongs to the stilbenes, natural phenolic phytoalexin metabolites elicited by abiotic stress (Chung et al., 2003). Resveratrol has also been observed in the root exudate metabolome of Quercus ilex under drought stress (Gargallo-Garriga et al., 2018), yet at very low concentrations since it was most likely readily degraded by bacteria (Kurt et al., 2018). In the present study, we detected resveratrol because probably we collected the exudates in axenic conditions suggesting hence its ecological role in the rhizosphere.

Interestingly, multiple stress exhibited a distinct and chemically diverse exudate profile compared to the individual stresses, with 13 metabolites being specifically up-exuded by maize root under combined stress (Table 1). Besides phenolics, which appeared as a main class of compounds generally modulated by stress, the combined stress triggered the specific exudation of tryptophan and palmitic acid. In this regard, it is known that the exudation of tryptophan could be stimulated by the plant beneficial Bacillus amyloliquefaciens (Liu et al., 2016). Moreover, the exudation of tryptophan-derived secondary metabolites are involved in the response to biotic stress (Baetz and Martinoia (2014). Analogously, palmitic acid was considered as metabolite recruiting beneficial rhizosphere bacteria in the halophyte Limonium sinense(Xiong et al, 2020) suggesting an ecological role in the soil salinization, an abiotic stress derived by a combination of salt, drought and heat. The specificity of exudation response to the combined stress, here observed, highlight the non-additive effects of the co-occurence of abiotic stresses. This is in agreement with previous findings observed for other maize traits such as root morphology (Vescio et al., 2021b), root microbiota (Vescio et al., 
2021a), leaf proteome (Zhao et al., 2016), and yield and nutrient uptake (Hussain et al., 2019). Our results, further, could be interesting considering that the exudation pattern probably was a depiction of what took place in the whole plant as pointed out by Gargallo-Garriga et al. (2018).

\section{Root exudation composition changed by root zone but not by root types}

Root exudation can change significantly along the root axis and different root types (Abenavoli et al. 2004; Tiziani et al. 2021). However, only the targeted analyses revealed significantly different metabolites in relation to the root zone. Aconitic acid, total phenols, total flavonoids, as well as glycine, alanine and threonine exudation has been significantly enhanced in maize apical root regions, compared to subapical ones (Figure 2). Generally, the root tip is considered to be very sensible to environmental stimuli (Baluška \& Mancuso 2013). Some studies suggested that the root tip functions as control center for sensing external nutrient concentrations, inducing alterations of root morphology and physiology (Baluška \& Mancuso 2013; Canarini, Kaiser, Merchant, Richter \& Wanek 2019; Tiziani et al.2020a). Moreover, the elongation zone, which in our study is located in the apical root zone, is known to be one of the physiologically most active root zones being a sort of command center for root responses to the environment (Baluska, Mancuso, Volkmann \& Barlow 2010; Baluška \& Mancuso 2013; Tiziani et al. 2020a). The lack of root type effects on the release of the exudates could be due to the restricted soil volume of the potting system, which may have limited the soil exploration of single root axes.

\section{Effect of root exudation on microbial community}

Roots determine the assembly and recruitment of plant-specific rhizosphere microbial communities by releasing exudates into the rhizosphere (Bulgarelli et al., 2013). Despite this important functional role in the plant-soil feedbacks, comprehensive and mechanistic evidence of the processes underlying the influence of root exudates towards rhizosphere microbiota is currently lacking. In this respect, rough correlations between the accumulation of root exudates and microbial communities (Chen et al., 2019) and few specific exudates included in soil (Badri et al., 2013; Zwetsloot et al., 2020; Gu et al., 2020) or growth medium (Zhalnina et al., 2018) indicated the effect(s) of root exudates on soil microbial community. Differently to these works, we applied a Multi-Omics Factor Analysis (MOFA+) using maize root exudome and microbiome dataset collected within the same growth unit to extract microbial taxa and maize root exudates that have a high probability to be involved in root-microbiota interaction.

The metabolites extracted were amino acids (L-serine, L-histidine, L-homocysteine), aminocyclopropane1-carboxilic acid, phenolics (p-coumaric, eugenol, quercetin 3- $O$-(6-acetyl-galactoside) 7- $O$-rhamnoside, rhamnetin and cinnamoyl glucose) together with carnosic acid (supplemental material 1). Amino acids in root exudates have been proposed as osmotically active compounds (Vives-Peris, Gómez-Cadenas \& PérezClemente 2018), whereas phenolics play a direct antioxidant activity and chemotaxis activity that has been proposed to shape the rhizosphere community (Iannucci et al. 2021; Lucini et al. 2019; Zuluaga et al. 2021). Except carnosic acid, all these compounds were negatively correlated with most gram-negative bacterial genus (supplemental material 1) suggesting that the maize root exudates finely modulated the depletion or enrichment of most diderm (or Gram negative) lineages. Interestingly, the pattern of soil microbial compositional shifts towards the gram-positive bacterial community is a universal response to abiotic stress (Xu and Coleman-Derr, 2019). On the other side, the increase of the abundance of specific bacteria belong to thePaenibacillus, Sphingomonas, Mesorhizobium andBacillus was also observed in our work (supplemental material 1) which, altough being gram-negative, have been associated with the plant tolerance to abiotic stress (Luo et al., 2019; Figueiredo, Burity, Martinez \& Chanway 2008; Khan, Mishra, Chauhan \& Nautiyal 2011; Liu, Sikora \& Park 2020b; Yadav, Yadav, Singh, Singh \& Singh 2021; Kang et al. 2019; Khan et al. 2020 ).

An interesting finding of our work was that the root exudates affecting bacterial taxa were stress-specific. In drought-stressed plants, we found that p-coumaric acid ethyl ester and L-serine negatively correlate with a pool of OTUs representing nine bacterial genera (supplementary material 1). P-coumaric acid belongs to the phenolic acids, a class of compounds that is well-known to affect the activity and diversity of the 
rhizosphere microbial communities. Indeed, this compound has been demonstrated to sharply affect the microbial community of the rhizosphere of crops (Zhou and Wu, 2012; Zhou et al., 2018) and specifically to reduce the abundance of Lysobacter (Folman et al. 2003), Haliangium (Fudou et al. 2001), and Gymnoascus spp. (Liu et al. 2017b). These species pointed out plant-growth-promoting and/or plant pathogen-inhibiting effects. In our study, we also found that L-serine negatively correlated with different bacterial genera. However, it has been also observed that amino acids as well as sugars showed a lower impact than phenolic acids on the microbial community (Badri et al., 2013). Hence, maize roots reducing the exudation of pcoumaric and L-serine in presence of drought stress determined from one side a lower soil accumulation of beneficial-microbial-inhibited metabolites (p-coumaric) and, at the same time, the preservation of an useful metabolite (L-serine) for the within-plant tolerance mechanisms. Indeed, we found that p-coumaric is negatively correlated withMucilaginibacter that has been found to alleviate salt stress (Fan, Subramanian \& Smith 2020), Sphingomonas andMesorhizobium which contributed to alleviate the effects of drought stress (Luo et al. , 2019; Yadav, Yadav, Singh, Singh \& Singh 2021) and Paenibacillus that are also well known to be recruited by plants during drought stress episodes (Figueiredo, Burity, Martinez \& Chanway 2008; Khan, Mishra, Chauhan \& Nautiyal 2011; Liu, Sikora \& Park 2020b).

In heat-stressed plants, we identified five compounds (1-aminocyclopropane-1-carboxilic, L-histidine, quercetin, eugenol, and rhamnetin) that explain most of the variation in the pattern of the root exudates, and that significantly correlate with microbial taxa (supplemental material 1). Also in this case, we observed an interaction between these compounds and several bacterial taxa that have been previously reported to have a beneficial influence on stressed plants. In particular, species of Bacillus are widely recognized to be beneficial microorganisms, helping plants to cope with a variety of stressors including heat (Kang et al. 2019; Khan et al.2020). While Chitinophaga, Mesorhizobium and Rhizobacter are all well-known plant-growth promoting rhizobacteria (Kour, Rana, Yadav \& Yadav 2019); no previous study reports a link betweenChitinophaga and abiotic stress.

Interestingly, in the combined-stressed plants, we identified three metabolites (L-homocysteine, cinnamoyl glucose and carnosic acid) that likely have a major role in the interaction between maize roots and soil microbiota and are completely different to that obtained in the single stress treatments (supplemental material 1). Similarly to what we observed for the single stressors, also these exudates correlated with taxa that have been previously reported to promote plant growth or alleviate the effect of plant stress. Among these genera, we observed (a) the gram negative Stenotrophomonas, including several species that have been reported as plant growth promoters and elicitors of plant resistance against biotic and abiotic stress (Singh and Jha, 2017), as well as the suppression of pathogens in the rhizosphere (Schmidt et al., 2012); (b) Microbacterium which improved the growth of sugarcane (Pereira et al., 2019) and pepper in presence of drought stress (Vílchez et al., 2018); (c) the Clavibacter that was observed in the rhizosphere of the halophyte Salicornia europaea (Hrynkiewicz et al., 2019); (d) the Dietzia whose specific strain, D. cinnamea 55, was isolated in abiotic stress environment and promoted the growth of maize plants (Khan et al., 2020) or the Dietzia natronolimnaea which improved the tolerance of the wheat plants to salt stress (Bharti et al., 2016).

Finally, within the same stress treatment, the identified metabolites interact with the same group of bacterial taxa (supplemental material 1) suggesting that, mechanistically, maize plants might modulate the exudation of a specific blend of molecules acting in concert to shape rhizosphere microbial communities.

Here we show that each individual stress (single and combined stress) produces a specific signature in the composition of root exudates targeting specific microbial taxa. In this respect, our results support the existence of a mechanism by which some root exudates are used by plants to recruit beneficial microorganisms (Naylor \& Coleman-Derr 2018; Williams \& de Vries 2020; Liu, Brettell, Qiu \& Singh 2020a). Root exudates are known to directly affect the availability of nutrients in the rhizosphere (Canarini, Merchant \& Dijkstra 2016). However, our findings indicate that indirect effects related to the need to recruit specific microbial communities is pivotal under drought, high temperature or their combination. In fact, our findings highlight a role of root exudates in the complex dynamics occurring in plant-microbe interactions during single or combined stress. The correlations observed confirm that specialized metabolites in root exudates may inter- 
fere with the mutualistic interactions between roots and defined phylogroups, as a well-known strategy to overcome stress conditions (O'Banion et al. 2020; Zuluaga et al. 2021). Edaphoclimatic conditions are known to determine plants' ability to exude compounds able to recruit and sustain a defined rhizosphere microbial community (Karlowsky etal. 2018) in a genotype-related manner (Iannucci et al. 2021). Notwithstanding, such modulation at rhizosphere community level is rather stress-specific and cannot be generalized, even across relatively related abiotic stresses.

\section{CONCLUSIONS}

Considering the fundamental role of root exudates in plant-soil interactions, we investigated the impact of single abiotic stresses, drought and heat, and their combination on the exudate profile of maize plants. Significantly different exudate profiles could be observed in presence of drought and heat stress, involving stress-specific up- and down-exuded compounds. Most likely, these root exudate blends could play a role in the plant acclimation processes induced by reduced water availability or increased soil temperature. The combination of drought and heat stress produced a different root exudate composition than single stresses, thus strengthening the idea that multiple stresses can interfere with plant processes in a non-additive way.

Taken together, our results highlight that root exudates are a pivotal process through which maize plants manage their rhizosphere processes in response to abiotic stresses, with distinctive traits being observed when maize plants were exposed to the combined stress. This fine tuning is also mechanistically linked to changes in soil microbial community composition and functioning. These new insights in rhizosphere processes could be useful to evaluate and define future mitigation strategies aimed at limiting the impact of drought and heatwaves on maize growth and productivity.

\section{ACKNOWLEDGEMENTS}

We are thankful to KWS Italy for providing the maize seeds. We also acknowledge funding from the $\mathrm{PhD}$ course "Scienze Agrarie, Alimentari e Forestali" of the University "Mediterranea" of Reggio Calabria and the Department AGRARIA at Università Mediterranea of Reggio Calabria for supporting Rosa Vescio. The authors thank the "Romeo ed Enrica Invernizzi" foundation (Milan, Italy) for its kind support to the metabolomics facility at Università Cattolica del Sacro Cuore. We would like to acknowledge support from the Consejería de Empleo, Universidades, Empresa y Medio Ambiente of the CARM, through the Fundación Séneca-Agencia de Ciencia y Tecnología de la Región de Murcia (Spain) (BM-M, Grant Number: 21252/PD/19). We thank the "Stiftung Südtiroler Sparkasse" for funding the PhD fellowship of Raphael Tiziani. This work was furthermore supported by grants from the Free University of Bolzano (NUMICS TN200E).

\section{REFERENCES}

Abenavoli M.R., Sorgonà A., Albano S. \& Cacco G. (2004) Coumarin Differentially Affects the Morphology of Different Root Types of Maize Seedlings. Journal of Chemical Ecology 30 , 1871-1883.

Alvarez S., Marsh E.L., Schroeder S.G. \& Schachtman D.P. (2008) Metabolomic and proteomic changes in the xylem sap of maize under drought. Plant, cell $\&$ environment $31,325-340$.

Argelaguet R., Velten B., Arnol D., Dietrich S., Zenz T., Marioni J.C., ... Stegle O. (2018) Multi-Omics Factor Analysis - a framework for unsupervised integration of multi-omics data sets. Molecular Systems Biology 14, e8124.

Ashraf M. \& Hafeez M. (2004) Thermotolerance of Pearl Millet and Maize at Early Growth Stages: Growth and Nutrient Relations. Biologia Plantarum 48, 81-86.

Astolfi S., Pii Y., Mimmo T., Lucini L., Miras-Moreno M.B., Coppa E., .. Cesco S. (2020) Single and combined fe and s deficiency differentially modulate root exudate composition in tomato: A double strategy for fe acquisition? International Journal of Molecular Sciences 21, 1-20. 
Atanassova M., Georgieva S. \& Ivancheva K. (2011) Total Phenolic and Total Flavonoid Contents , Antioxidant Capacity and Biological Contaminants in Medicinal Herbs. Journal of the University of Chemical Technology and Metallurgy, 46 , 81-88.

Badri D V., Chaparro J M., Zhang R, Shen Q, Vivanco J M. 2013 Application of Natural Blends of Phytochemicals Derived from the Root Exudates of Arabidopsis to the Soil Reveal That Phenolic-related Compounds Predominantly Modulate the Soil Microbiome*, Journal of Biological Chemistry, Volume 288, Issue 7, Pages 4502-4512.

Baetz, U., \& Martinoia, E. (2014). Root exudates: the hidden part of plant defense. Trends in Plant Science , $19,90-98$.

Baluška F. \& Mancuso S. (2013) Root Apex Transition Zone As Oscillatory Zone . Frontiers in Plant Science 4, 354.

Baluska F., Mancuso S., Volkmann D. \& Barlow P.W. (2010) Root apex transition zone: a signalling-response nexus in the root. Trends in plant science 15 , 402-408.

Barnawal D., Bharti N., Maji D., Chanotiya C.S. \& Kalra A. (2012) 1-Aminocyclopropane-1-carboxylic acid (ACC) deaminase-containing rhizobacteria protect Ocimum sanctum plants during waterlogging stress via reduced ethylene generation. Plant physiology and biochemistry : PPB 58,227-235.

Belt K., Huang S., Thatcher L.F., Casarotto H., Singh K.B., Van Aken O. \& Millar A.H. (2017) Salicylic Acid-Dependent Plant Stress Signaling via Mitochondrial Succinate Dehydrogenase. Plant physiology173, 2029-2040.

Bengough G. (2003) Plant Roots: The Hidden Half. 3rd Edition. Edited by Y. Waisel, A. Eshel and U. Kafkafi. Monticello, NY, USA: Marcel Dekker Inc. (2002), pp. 1120, US\$250.00. ISBN 0-8247-06315.Experimental Agriculture 39 , 219-222.

Bharti, N., Pandey, S., Barnawal, D. et al. Plant growth promoting rhizobacteria Dietzia natronolimnaea modulates the expression of stress responsive genes providing protection of wheat from salinity stress. Sci Rep 6, 34768 (2016). https://doi.org/10.1038/srep34768

Bokhari, A., Essack, M., Lafi, F.F. et al. Bioprospecting desert plant Bacillus endophytic strains for their potential to enhance plant stress tolerance. Sci Rep 9, 18154 (2019). https://doi.org/10.1038/s41598-019$54685-\mathrm{y}$

Bouain N., Krouk G., Lacombe B. \& Rouached H. (2019) Getting to the Root of Plant Mineral Nutrition: Combinatorial Nutrient Stresses Reveal Emergent Properties. Trends in Plant Science 24, 542-552.

Bulgarelli, D., Schlaeppi, K., Spaepen, S., Ver Loren van Themaat, E. \& Schulze-Lefert, P. Structure and functions of the bacterial microbiota of plants. Annu. Rev. Plant. Biol. 64, 807-838 (2013).

Canarini A., Kaiser C., Merchant A., Richter A. \& Wanek W. (2019) Root exudation of primary metabolites: Mechanisms and their roles in plant responses to environmental stimuli. Frontiers in Plant Science10 , 157.

Canarini A., Merchant A. \& Dijkstra F.A. (2016) Drought effects on Helianthus annuus and Glycine max metabolites: from phloem to root exudates. Rhizosphere $\mathbf{2}, 85-97$.

Chen, S., Waghmode, T.R., Sun, R. et al. Root-associated microbiomes of wheat under the combined effect of plant development and nitrogen fertilization. Microbiome 7, 136 (2019). https://doi.org/10.1186/s40168019-0750-2

Chung IM, Park MR, Chun JC, Yun SJ. 2003. Resveratrol accumulation and resveratrol synthase gene expression in response to abiotic stresses and hormones in peanut plants. Plant Sci 164:103109.https://doi.org/10.1016/S0168-9452(02)00341-2 
Czarnota M.A., Rimando A.M. \& Weston L.A. (2003) Evaluation of Root Exudates of Seven Sorghum Accessions. Journal of Chemical Ecology29 , 2073-2083.

Doornbos R.F., van Loon L.C. \& Bakker P.A.H.M. (2012) Impact of root exudates and plant defense signaling on bacterial communities in the rhizosphere. A review. Agronomy for Sustainable Development32 , 227-243.

Dutta S., Mohanty S. \& Tripathy B.C. (2009) Role of Temperature Stress on Chloroplast Biogenesis and Protein Import in Pea. Plant Physiology 150 , 1050 LP - 1061.

El-Tarabily K.A. (2008) Promotion of tomato (Lycopersicon esculentum Mill.) plant growth by rhizosphere competent 1-aminocyclopropane-1-carboxylic acid deaminase-producing streptomycete actinomycetes. Plant and Soil $308,161-174$.

Enebe MC, Babalola OO. The influence of plant growth-promoting rhizobacteria in plant tolerance to abiotic stress: a survival strategy. Appl Microbiol Biotechnol. 2018 Sep;102(18):7821-7835. doi: 10.1007/s00253018-9214-z.

Fahad S., Bajwa A.A., Nazir U., Anjum S.A., Farooq A., Zohaib A., ... Huang J. (2017) Crop production under drought and heat stress: Plant responses and management options. Frontiers in Plant Science $\mathbf{8}$, $1-16$.

Fan D., Subramanian S. \& Smith D.L. (2020) Plant endophytes promote growth and alleviate salt stress in Arabidopsis thaliana.Scientific Reports 10, 12740.

Figueiredo M., Burity H., Martinez C. \& Chanway C. (2008) Alleviation of drought stress in the common bean (Phaseolus vulgaris L.) by co-inoculation with Paenibacillus polymyxa and Rhizobium tropici.Applied soil ecology : a section of Agriculture, Ecosystems Ëamp; Environment 40 , 182-188.

García-Pérez P., Miras-Moreno B., Lucini L. \& Gallego P.P. (2021) The metabolomics reveals intraspecies variability of bioactive compounds in elicited suspension cell cultures of three Bryophyllum species.Industrial Crops and Products 163, 113322.

Gargallo-Garriga A., Preece C., Sardans J., Oravec M., Urban O. \& Peñuelas J. (2018) Root exudate metabolomes change under drought and show limited capacity for recovery. Scientific Reports8 , 12696.

Gelsomino A., Tortorella D., Cianci V., Petrovičová B., Sorgonà A., Piccolo A. \& Rosa Abenavoli M. (2010) Effects of a biomimetic iron-porphyrin on soil respiration and maize root morphology as by a microcosm experiment. Journal of Plant Nutrition and Soil Science173 , 399-406.

Gharibi S., Sayed Tabatabaei B.E., Saeidi G., Talebi M. \& Matkowski A. (2019) The effect of drought stress on polyphenolic compounds and expression of flavonoid biosynthesis related genes in Achillea pachycephala Rech.f. Phytochemistry 162, 90-98.

Gu, Y, Wang, X, Yang, T, et al. Chemical structure predicts the effect of plant-derived low-molecular weight compounds on soil microbiome structure and pathogen suppression. Funct Ecol. 2020; 34: 21582169. https://doi.org/10.1111/1365-2435.13624

Harbort C.J., Hashimoto M., Inoue H., Niu Y., Guan R., Rombola‘ A.D., Kopriva S., Voges M. J.E.E.E., Sattely E. S., Garrido-Oter R., Schulze-Lefert P. 2020. Root-Secreted Coumarins and the Microbiota Interact to Improve Iron Nutrition in Arabidopsis. Cell Host $\&$ Microbe . 28 , 825-837

Heckathorn S.A., Giri A., Mishra S. \& Bista D. (2013) Heat Stress and Roots. Climate Change and Plant Abiotic Stress Tolerance, 109-136.

Henry A., Doucette W., Norton J. \& Bugbee B. (2007) Changes in crested wheatgrass root exudation caused by flood, drought, and nutrient stress. Journal of environmental quality 36 , 904-912.

Hrynkiewicz K., Patz S., Ruppel S. (2019) Salicornia europaea L. as an underutilized saline-tolerant plant inhabited by endophytic diazotrophs.J. Adv. Res., pp. 49-56, 10.1016/j.jare.2019.05.002 
Hussain, H.A., Men, S., Hussain, S. et al. Interactive effects of drought and heat stresses on morphophysiological attributes, yield, nutrient uptake and oxidative status in maize hybrids. Sci Rep 9, 3890 (2019). https://doi.org/10.1038/s41598-019-40362-

Iannucci, A., Canfora, L., Nigro, F., De Vita, P., \& Beleggia, R. (2021). Relationships between root morphology, root exudate compounds and rhizosphere microbial community in durum wheat. Applied Soil Ecology , $158,103781$.

Iijima, M., Griffiths, B., \& Bengough, A.G. (2000) Sloughing of cap cells and carbon exudation from maize seedling roots in compacted sand. New Phytol.145(3):477-482. doi: 10.1046/j.1469-8137.2000.00595.x.

Jaeger C.H. 3rd, Lindow S.E., Miller W., Clark E. \& Firestone M.K. (1999) Mapping of sugar and amino acid availability in soil around roots with bacterial sensors of sucrose and tryptophan. Applied and environmental microbiology 65, 2685-2690.

Kang S.-M., Khan A.L., Waqas M., Asaf S., Lee K.-E., Park Y.-G., . . Lee I.-J. (2019) Integrated phytohormone production by the plant growth-promoting rhizobacterium Bacillus tequilensis SSB07 induced thermotolerance in soybean. Journal of Plant Interactions14, 416-423.

Karlowsky S., Augusti A., Ingrisch J., Akanda M.K.U., Bahn M. \& Gleixner G. (2018) Drought-Induced Accumulation of Root Exudates Supports Post-drought Recovery of Microbes in Mountain Grassland .Frontiers in Plant Science $\mathbf{9}, 1593$.

Karst J., Gaster J., Wiley E. \& Landhäusser S.M. (2017) Stress differentially causes roots of tree seedlings to exude carbon. Tree Physiology $37,154-164$.

Khan M.A., Asaf S., Khan A.L., Jan R., Kang S.-M., Kim K.-M. \& Lee I.-J. (2020) Thermotolerance effect of plant growth-promoting Bacillus cereus SA1 on soybean during heat stress. BMC Microbiology20 , 175.

Khan N., Mishra A., Chauhan P.S. \& Nautiyal C.S. (2011) Induction of Paenibacillus lentimorbus biofilm by sodium alginate and $\mathrm{CaCl} 2$ alleviates drought stress in chickpea. Annals of Applied Biology159, 372-386.

Khan N, Martínez-Hidalgo P, Humm EA, Maymon M, Kaplan D and Hirsch AM (2020) Inoculation With a Microbe Isolated From the Negev Desert Enhances Corn Growth. Front. Microbiol. 11:1149. doi: 10.3389/fmicb.2020.01149

Khorassani R., Hettwer U., Ratzinger A., Steingrobe B., Karlovsky P. \& Claassen N. (2011) Citramalic acid and salicylic acid in sugar beet root exudates solubilize soil phosphorus. BMC plant biology11, 121.

Kour D., Rana K.L., Yadav N. \& Yadav A.N. (2019) Plant Growth Promoting Rhizobacteria for Agricultural Sustainability .

Król A., Amarowicz R. \& Weidner S. (2014) Changes in the composition of phenolic compounds and antioxidant properties of grapevine roots and leaves (Vitis vinifera L.) under continuous of long-term drought stress.Acta Physiologiae Plantarum 36 , 1491-1499.

Kurt Z, Minoia M, Spain JC. Resveratrol as a Growth Substrate for Bacteria from the Rhizosphere. Appl Environ Microbiol. 2018 May 1;84(10):e00104-18. doi: 10.1128/AEM.00104-18. PMID: 29523548; PMCID: PMC5930378.

Li J. \& Copeland L. (2000) Role of malonate in chickpeas.Phytochemistry 54, 585-589.

Li Y, Peng Q, Selimi D, Wang Q, Charkowski AO, Chen X, Yang CH. The plant phenolic compound pcoumaric acid represses gene expression in the Dickeya dadantii type III secretion system. Appl Environ Microbiol. 2009 Mar;75(5):1223-8. doi: 10.1128/AEM.02015-08

Liu H., Brettell L.E., Qiu Z. \& Singh B.K. (2020a) Microbiome-Mediated Stress Resistance in Plants. Trends in Plant Science 25, 733-743. 
Liu W., Sikora E. \& Park S.-W. (2020b) Plant growth-promoting rhizobacterium, Paenibacillus polymyxa CR1, upregulates dehydration-responsive genes, RD29A and RD29B, during priming drought tolerance in arabidopsis. Plant physiology and biochemistry : PPB 156, 146-154.

Liu, Y., Chen, L., Zhang, N., Li, Z., Zhang, G., Xu, Y., Shen, Q \& Zhang, R. (2016). Plant-microbe communication enhances auxin biosynthesis by a root-associated bacterium, Bacillus amyloliquefaciens SQR9. Molecular Plant-Microbe Interactions , 29 , 324-330.

Liu, Y., Li, X., Cai, K., Cai, L., Lu, N., \& Shi, J. (2015). Identification of benzoic acid and 3-phenylpropanoic acid in tobacco root exudates and their role in the growth of rhizosphere microorganisms. Applied Soil Ecology , $93,78-87$.

Love M.I., Huber W. \& Anders S. (2014) Moderated estimation of fold change and dispersion for RNA-seq data with DESeq2. Genome Biology 15, 550.

Lucini L., Colla G., Miras Moreno M.B., Bernardo L., Cardarelli M., Terzi V., .. Rouphael Y. (2019) Inoculation of Rhizoglomus irregulare or Trichoderma atroviride differentially modulates metabolite profiling of wheat root exudates. Phytochemistry 157, 158-167.

Luo Y., Wang F., Huang Y., Zhou M., Gao J., Yan T., ... An L. (2019) Sphingomonas sp. Cra20 Increases Plant Growth Rate and Alters Rhizosphere Microbial Community Structure of Arabidopsis thaliana Under Drought Stress . Frontiers in Microbiology 10, 1221.

Lupini A., Sorgona A., Princi M.P., Sunseri F. \& Abenavoli M.R. (2016) Morphological and physiological effects of trans-cinnamic acid and its hydroxylated derivatives on maize root types. Plant Growth Regulation $\mathbf{7 8}, 263-273$.

Lynch J.P. (2013) Steep, cheap and deep: an ideotype to optimize water and N acquisition by maize root systems. Annals of Botany $112,347-357$.

Marastoni, L., Lucini, L., Miras-Moreno, B. et al. Changes in physiological activities and root exudation profile of two grapevine rootstocks reveal common and specific strategies for Fe acquisition. Sci Rep 10, 18839 (2020). https://doi.org/10.1038/s41598-020-75317-w

Mathesius U. (2001) Flavonoids induced in cells undergoing nodule organogenesis in white clover are regulators of auxin breakdown by peroxidase. Journal of Experimental Botany 52 , 419-426.

McCully M.E. \& Canny M.J. (1985) Localisation of translocated 14C in roots and root exudates of fieldgrown maize. Physiologia Plantarum 65, 380-392.

Meseka S., Menkir A., Bossey B. \& Mengesha W. (2018) Performance Assessment of Drought Tolerant Maize Hybrids under Combined Drought and Heat Stress. Agronomy 8 .

Mickelsen O. \& Yamamoto R.S. (1958) Methods for the Determination of Thiamine. Methods of Biochemical Analysis , 191-257.

Miliauskas G., Venskutonis P.R. \& van Beek T.A. (2004) Screening of radical scavenging activity of some medicinal and aromatic plant extracts. Food Chemistry 85, 231-237.

Mimmo T., Pii Y., Valentinuzzi F., Astolfi S., Lehto N., Robinson B., .. Cesco S. (2018) Nutrient availability in the rhizosphere: A review. Acta Horticulturae 1217, 13-27.

Moe L.A. (2013) Amino acids in the rhizosphere: From plants to microbes.American Journal of Botany 100 , 1692-1705.

Mommer L., Hinsinger P., Prigent-Combaret C. \& Visser E.J.W. (2016) Advances in the rhizosphere: stretching the interface of life.Plant and Soil 407, 1-8. 
Muthusamy M., Uma S., Backiyarani S., Saraswathi M.S. \& Chandrasekar A. (2016) Transcriptomic Changes of Drought-Tolerant and Sensitive Banana Cultivars Exposed to Drought Stress . Frontiers in Plant Science 7 , 1609 .

Naylor D. \& Coleman-Derr D. (2018) Drought Stress and Root-Associated Bacterial Communities . Frontiers in Plant Science 8, 2223.

Nishiyama Y. \& Murata N. (2014) Revised scheme for the mechanism of photoinhibition and its application to enhance the abiotic stress tolerance of the photosynthetic machinery. Applied microbiology and biotechnology $98,8777-8796$.

O'Banion, B. S., O'Neal, L., Alexandre, G., \& Lebeis, S. L. (2020). Bridging the gap between single-strain and community-level plant-microbe chemical interactions. Molecular Plant-Microbe Interactions ,33 , 124134.

Obata T., Witt S., Lisec J., Palacios-Rojas N., Florez-Sarasa I., Yousfi S., . . Fernie A.R. (2015) Metabolite profiles of maize leaves in drought, heat, and combined stress field trials reveal the relationship between metabolism and grain yield. Plant Physiology 169, 2665-2683.

Oburger E. \& Jones D.L. (2018) Sampling root exudates - Mission impossible? Rhizosphere 6 , 116-133.

Olanrewaju OS, Glick BR, Babalola OO. Mechanisms of action of plant growth promoting bacteria. World J Microbiol Biotechnol. 2017;33(11):197. Published 2017 Oct 6. doi:10.1007/s11274-017-2364-9

Pandey P., Ramegowda V. \& Senthil-Kumar M. (2015) Shared and unique responses of plants to multiple individual stresses and stress combinations: physiological and molecular mechanisms. Frontiers in Plant Science $6,723$.

Parvin K., Hasanuzzaman M., Bhuyan M.H.M.B., Mohsin S.M. \& Fujita M. (2019) Quercetin Mediated Salt Tolerance in Tomato through the Enhancement of Plant Antioxidant Defense and Glyoxalase Systems.Plants 8 .

Pereira LB, Andrade GS, Meneghin SP, Vicentini R, Ottoboni LMM. Prospecting Plant Growth-Promoting Bacteria Isolated from the Rhizosphere of Sugarcane Under Drought Stress. Curr Microbiol. 2019 Nov;76(11):1345-1354. doi: 10.1007/s00284-019-01749-x

Pii Y., Penn A., Terzano R., Crecchio C., Mimmo T. \& Cesco S. (2015) Plant-microorganism-soil interactions influence the Fe availability in the rhizosphere of cucumber plants. Plant Physiology and Biochemistry 87 , $45-52$.

Preece C., Farre-Armengol G., Llusia J. \& Penuelas J. (2018) Thirsty tree roots exude more carbon. Tree Physiology 38, 690-695.

Rivero R.M., Mestre T.C., Mittler R., Rubio F., Garcia-Sanchez F. \& Martinez V. (2014) The combined effect of salinity and heat reveals a specific physiological, biochemical and molecular response in tomato plants. Plant, cell \& environment 37 , 1059-1073.

Thimmaraju Rudrappa, Kirk J. Czymmek, Paul W. Pare, Harsh P. Bais, Root-Secreted Malic Acid Recruits Beneficial Soil Bacteria, Plant Physiology, Volume 148, Issue 3, November 2008, Pages 1547-1556, https://doi.org/10.1104/pp.108.127613

Rubio G., Sorgonà A. \& Lynch J.P. (2004) Spatial mapping of phosphorus influx in bean root systems using digital autoradiography. Journal of experimental botany 55 , 2269-2280.

Schmidt, C.S., Alavi, M., Cardinale, M. et al. Stenotrophomonas rhizophila DSM14405T promotes plant growth probably by altering fungal communities in the rhizosphere. Biol Fertil Soils 48, 947-960 (2012). https://doi.org/10.1007/s00374-012-0688-z 
Singh RP, Jha P N. The PGPR Stenotrophomonas maltophilia SBP-9 augments resistance against biotic and abiotic stress in wheat plants. Frontiers in Microbiology, 2017, 8, 1945 10.3389/fmicb.2017.01945

Sorgonà A., Lupini A., Mercati F., Di Dio L., Sunseri F. \& Abenavoli M.R. (2011) Nitrate uptake along the maize primary root: an integrated physiological and molecular approach. Plant, cell 83 environment 34 , $1127-1140$.

Stringlis I. A, de Jonge R., Pieterse C. M. J. (2019) The Age of Coumarins in Plant-Microbe Interactions. Plant and Cell Physiology, Volume 60, Issue 7, Pages 1405-1419, https://doi.org/10.1093/pcp/pcz076

Thomason K., Babar M.A., Erickson J.E., Mulvaney M., Beecher C. \& MacDonald G. (2018) Comparative physiological and metabolomics analysis of wheat (Triticum aestivum L.) following post-anthesis heat stress.PLOS ONE 13 , e0197919.

Tiwari G., Duraivadivel P., Sharma S. \& P. H. (2018) 1-Aminocyclopropane-1-carboxylic acid deaminase producing beneficial rhizobacteria ameliorate the biomass characters of Panicum maximum Jacq. by mitigating drought and salt stress. Scientific Reports8, 17513.

Tiziani R., Mimmo T., Valentinuzzi F., Pii Y., Celletti S. \& Cesco S. (2020a) Root Handling Affects Carboxylates Exudation and Phosphate Uptake of White Lupin Roots . Frontiers in Plant Science11, 1403.

Tiziani R., Pii Y., Celletti S., Cesco S. \& Mimmo T. (2020b) Phosphorus deficiency changes carbon isotope fractionation and triggers exudate reacquisition in tomato plants. Scientific Reports $\mathbf{1 0}$.

Tiziani R., Puschenreiter M., Smolders E., Mimmo T., Herrera J.C., Cesco S. \& Santner J. (2021) Millimetreresolution mapping of citrate exuded from soil-grown roots using a novel, low-invasive sampling technique. Journal of Experimental Botany 72 , 3513-3525.

Tsugawa H., Cajka T., Kind T., Ma Y., Higgins B., Ikeda K., .. A Arita M. (2015) MS-DIAL: data-independent MS/MS deconvolution for comprehensive metabolome analysis. Nature Methods 12 , 523-526.

Ullah N., Yüce M., Neslihan Öztürk Gökçe Z. \& Budak H. (2017) Comparative metabolite profiling of drought stress in roots and leaves of seven Triticeae species. BMC Genomics 18, 1-12.

Valentinuzzi F., Mimmo T., Cesco S., Al Mamun S., Santner J., Hoefer C., ... Lehto N. (2015) The effect of lime on the rhizosphere processes and elemental uptake of white lupin. Environmental and Experimental Botany 118, 85-94.

Venkatesh, J., \& Park, S. W. (2014). Role of L-ascorbate in alleviating abiotic stresses in crop plants. Botanical studies, 55(1), 38. https://doi.org/10.1186/1999-3110-55-38

Vescio R., Malacrinò A., Bennett A.E. \& Sorgonà A. (2021a) Single and combined abiotic stressors affect maize rhizosphere bacterial microbiota. Rhizosphere 17, 100318.

Vescio R. et al. (2021b) Single and combined abiotic stress in maize root morphology. Plants, 10(1), 5. https://doi.org/10.3390/plants10010005

Vílchez JI, Niehaus K, Dowling DN, González-López J and Manzanera M (2018) Protection of Pepper Plants from Drought by Microbacterium sp. 3J1 by Modulation of the Plant's Glutamine and $\alpha$-ketoglutarate Content: A Comparative Metabolomics Approach. Front. Microbiol. 9:284. doi: 10.3389/fmicb.2018.00284

Vives-Peris, V., Gómez-Cadenas, A., \& Pérez-Clemente, R. M. (2018). Salt stress alleviation in citrus plants by plant growth-promoting rhizobacteria Pseudomonas putida and Novosphingobium sp.Plant Cell Reports , 37, 1557-1569.

Vives-Peris V., López-Climent M.F., Pérez-Clemente R.M. \& Gómez-Cadenas A. (2020) Root Involvement in Plant Responses to Adverse Environmental Conditions. Agronomy 10 .

Vives-Peris V., de Ollas C., Gómez-Cadenas A. \& Pérez-Clemente R.M. (2019) Root exudates: from plant to rhizosphere and beyond. Plant Cell Reports . 
de Vries F.T., Griffiths R.I., Bailey M., Craig H., Girlanda M., Gweon H.S., .. Bardgett R.D. (2018) Soil bacterial networks are less stable under drought than fungal networks. Nature Communications 9 , 3033.

Wang S., Zhou D., Shi M., Feng H., Xie X., Sun P., ... Zhao J. (2021) Expression patterns of four key genes involved in strawberry eugenol synthesis under abiotic stresses. Acta Ecologica Sinica .

Weidner S., Karolak M., Karamać M., Kosińska A. \& Amarowicz R. (2009) Phenolic compounds and properties of antioxidants in grapevine roots (vitis vinifera 1.) under drought stress followed by recovery.Acta Societatis Botanicorum Poloniae 78, 97-103.

Williams A. \& de Vries F.T. (2020) Plant root exudation under drought: implications for ecosystem functioning. New Phytologist225, 1899-1905.

Xu L, Coleman-Derr D, 2019 Causes and consequences of a conserved bacterial root microbiome response to drought stress. Current Opinion in Microbiology,Volume 49,Pages 1-6

Yadav A., Yadav A., Singh R.P., Singh A.L. \& Singh M. (2021) Identification of genes involved in phosphate solubilization and drought stress tolerance in chickpea symbiont Mesorhizobium ciceri Ca181.Archives of microbiology 203, 1167-1174.

Yaqoob S., Bhatti H.N., Sultana B. \& Shahid M. (2020) Prognosticating the potential of sorghum bicolor root exudates in response to abiotic stress. Pakistan Journal of Agricultural Sciences 57 , 1661-1668.

Yu J., Du H., Xu M. \& Huang B. Metabolic Responses to Heat Stress under Elevated Atmospheric CO2 Concentration in a Cool-season Grass Species. Journal of the American Society for Horticultural Science J. Amer. Soc. Hort. Sci. 137 , 221-228.

Xiong YW, Li XW, Wang TT, Gong Y, Zhang CM, Xing K, Qin S. Root exudates-driven rhizosphere recruitment of the plant growth-promoting rhizobacterium Bacillus flexus KLBMP 4941 and its growthpromoting effect on the coastal halophyte Limonium sinense under salt stress. Ecotoxicol Environ Saf. 2020 May;194:110374. doi: 10.1016/j.ecoenv.2020.110374. Epub 2020 Feb 28. PMID: 32120174.

Zandalinas S.I., Mittler R., Balfagón D., Arbona V. \& Gómez-Cadenas A. (2018) Plant adaptations to the combination of drought and high temperatures. Physiologia Plantarum 162, 2-12.

Zhalnina, K., Louie, K.B., Hao, Z. et al. Dynamic root exudate chemistry and microbial substrate preferences drive patterns in rhizosphere microbial community assembly. Nat Microbiol 3, 470-480 (2018). https://doi.org/10.1038/s41564-018-0129-3

Zhan A., Schneider H. \& Lynch J.P. (2015) Reduced Lateral Root Branching Density Improves Drought Tolerance in Maize. Plant physiology $168,1603-1615$.

Zhang J., Chen G., Zhao P., Zhou Q. \& Zhao X. (2017) The abundance of certain metabolites responds to drought stress in the highly drought tolerant plant Caragana korshinskii. Acta Physiologiae Plantarum39 .

Zhang J., Yang D., Li M. \& Shi L. (2016) Metabolic Profiles Reveal Changes in Wild and Cultivated Soybean Seedling Leaves under Salt Stress. PLOS ONE 11, e0159622.

Zhao F, Zhang D, Zhao Y, Wang W, Yang H, Tai F, Li C, Hu X (2016) The difference of physiological and proteomic changes in maize leaves adaptation to drought, heat, and combined both stresses. Front Plant Sci. 7: 1471.

Zhou X, Wu F. p-Coumaric acid influenced cucumber rhizosphere soil microbial communities and the growth of Fusarium oxysporum f.sp. cucumerinum Owen. PLoS One. 2012;7(10):e48288. doi:10.1371/journal.pone.0048288

Zhou, X., Zhang, J., Pan, D. et al. p-Coumaric can alter the composition of cucumber rhizosphere microbial communities and induce negative plant-microbial interactions. Biol Fertil Soils 54, 363-372 (2018). https://doi.org/10.1007/s00374-018-1265-x 
Zuluaga, M. Y. A., Milani, K. M. L., Miras-Moreno, B., Lucini, L., Valentinuzzi, F., Mimmo, T., Pii, Y., Cesco, S., Pains Rodrigues, E., de Oliveira, A. L. M. (2021). Inoculation with plant growth-promoting bacteria alters the rhizosphere functioning of tomato plants.Applied Soil Ecology , 158 , 103784.

Zwetsloot MJ., Muñoz Ucros J, Wickings K, Wilhelm R C., Sparks J, Buckley D H., Bauerle T L., 2020 Prevalent root-derived phenolics drive shifts in microbial community composition and prime decomposition in forest soil, Soil Biology and Biochemistry, Volume 145, 107797,

Table 1. Significant metabolites specifically exuded in the presence of drought, heat and/or combined stress.

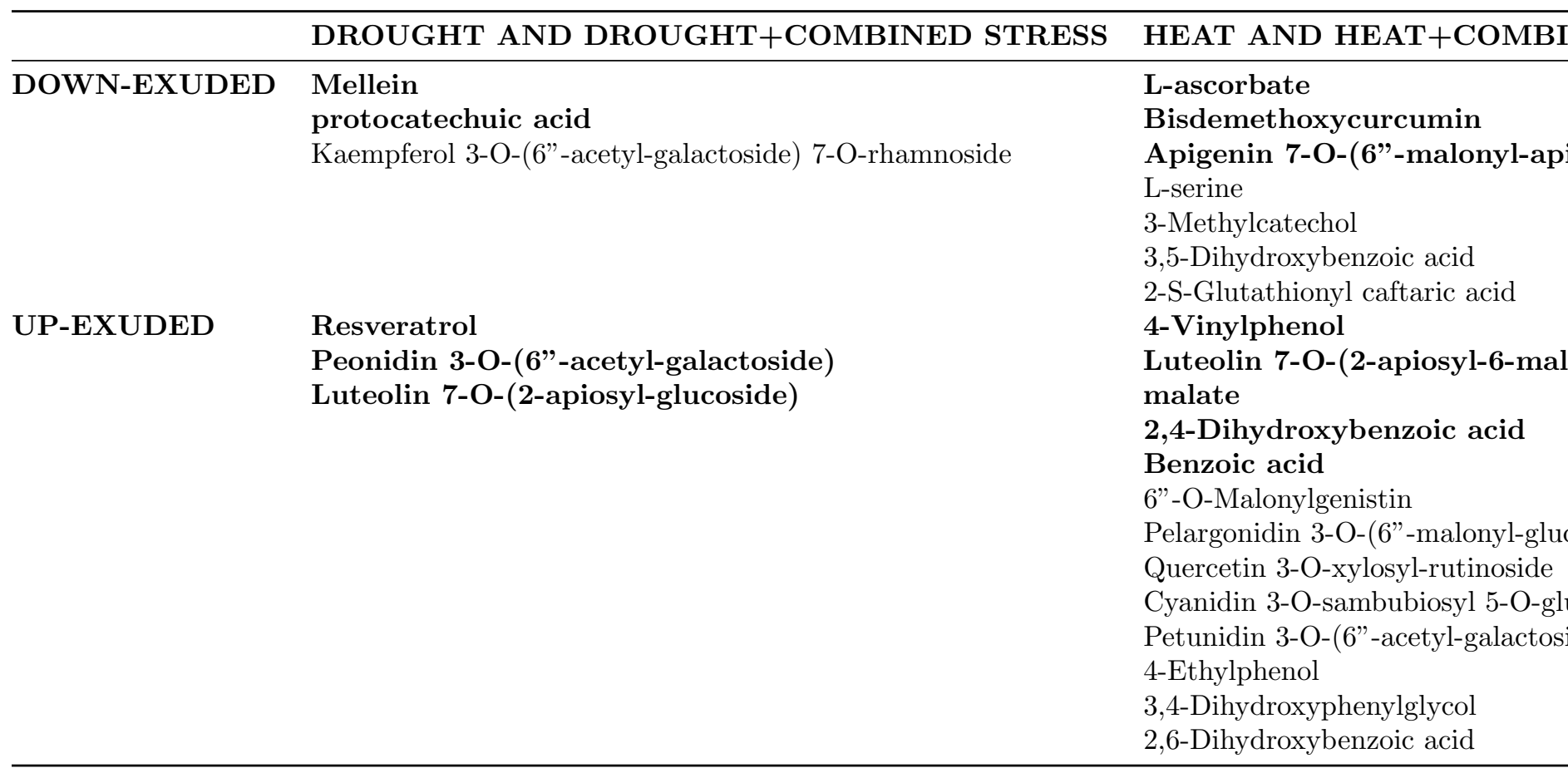

Metabolites in bold type indicate their modulation exclusively in the presence of the single stress or the combined stress.

Metabolites in normal type indicate their modulation shared between the single (drought and heat) and combined stress

Figure 1. Scheme (A) and picture of the compartmented chambers (B) and the sampling method used for the collection of the maize root exudates $(\mathrm{C})$.

Figure 2 . Compounds detected in the maize root exudate collection solution: total phenols (a), total flavonols (b) total flavonoids (c), aconitic acid (d), alanine (e), glycine (f), threonine (g). Plants have been grown under following conditions: control (T1: $25^{\circ} \mathrm{C}, 80 \%$ soil field capacity); drought $\left(\mathrm{T} 2: 25^{\circ} \mathrm{C}\right.$, $30 \%$ soil field capacity); heat (T3: $32^{\circ} \mathrm{C}, 80 \%$ soil field capacity); combined stress (T4: $32^{\circ} \mathrm{C} 30 \%$ soil field capacity). The exudates are collected to the following root type-zone: seminal-apical (), =, seminal-subapical (), primary-apical (), primary-subapical (). The values are reported as means +- standard errors. Three way ANOVA with Tukey post hoc test: ${ }^{*} p=0.01-0.05,{ }^{* *} \mathrm{p}=0.01-0.001 ;{ }^{* *} p<0.001$.

Figure 3. Unsupervised hierarchical cluster analysis (Euclidean distance; linkage rule: Ward) carried out from root exudates chemical profiles. Metabolites were obtained by UHPLC-ESI/QTOF-MS untargeted analysis, and their intensities used to build up the fold-change heatmap here provided. 
Figure 4. Score plot of Orthogonal projection to latent structures discriminant analysis (OPLS-DA) supervised modelling carried out from untargeted metabolomics profiles of root exudates (correlation $R^{2} Y=$ $0.83)$, prediction ability $\left.\mathrm{Q}^{2} \mathrm{Y}=0.48\right)$.

Figure 5 . Venn diagram summarizing the metabolites differentially up-exuded (A) and down-exuded (B) that resulted from the volcano analysis.

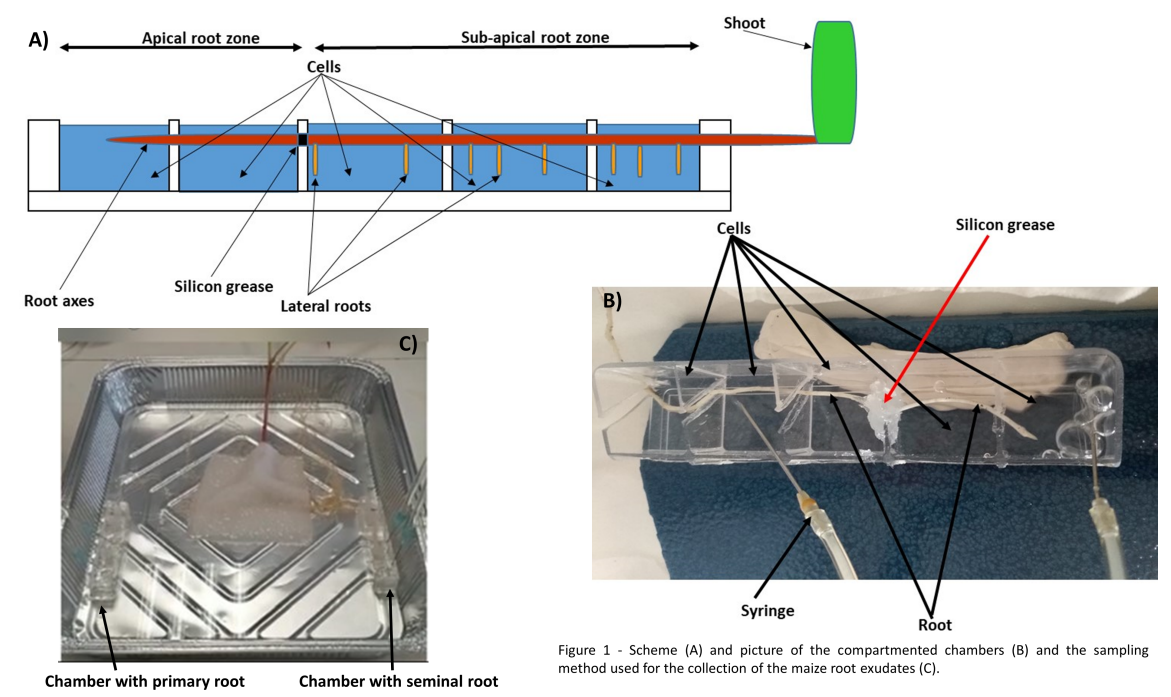



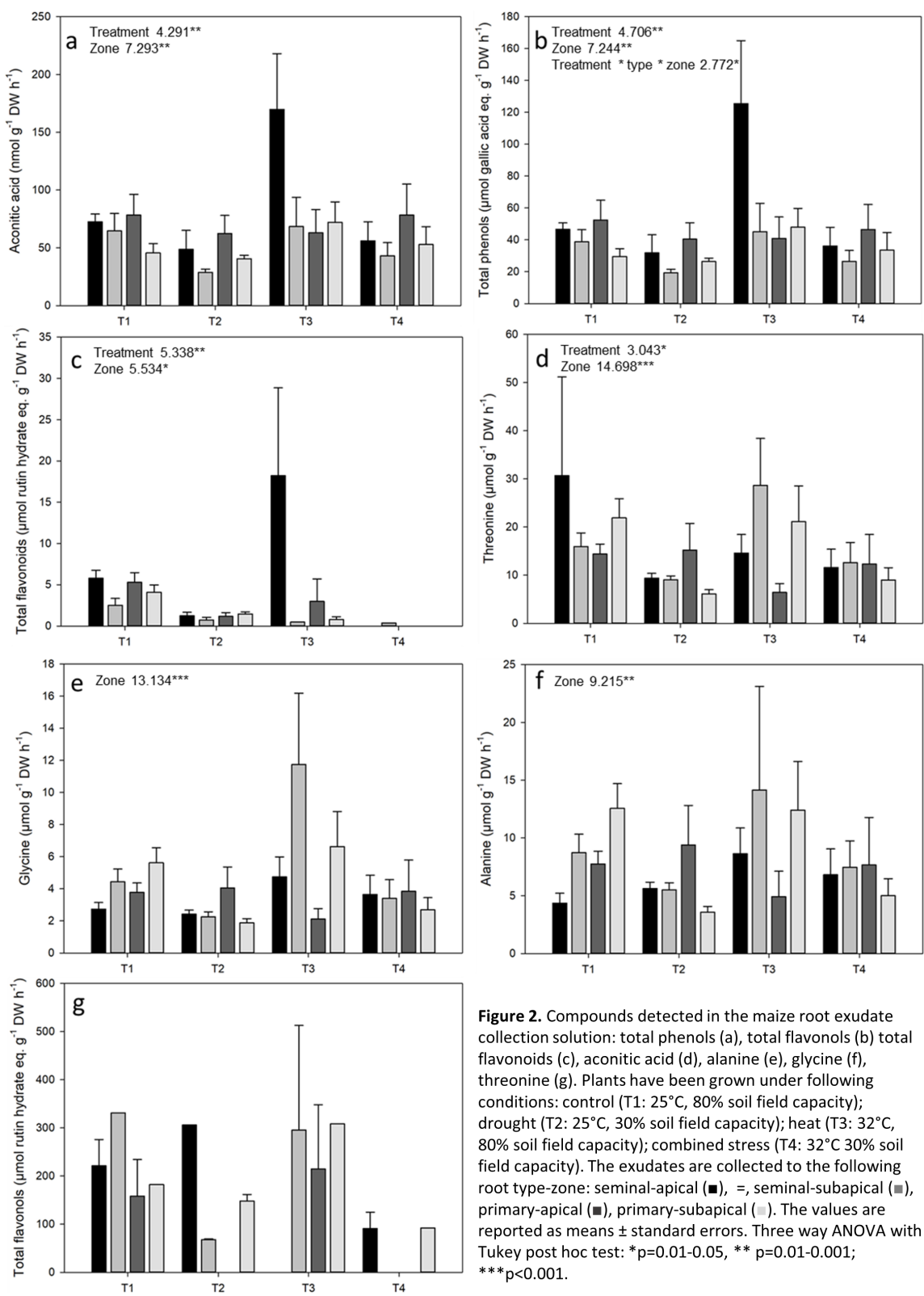

Figure 2. Compounds detected in the maize root exudate collection solution: total phenols (a), total flavonols (b) total flavonoids (c), aconitic acid (d), alanine (e), glycine (f),

threonine $(\mathrm{g})$. Plants have been grown under following

conditions: control (T1: $25^{\circ} \mathrm{C}, 80 \%$ soil field capacity);

drought $\left(\mathrm{T} 2: 25^{\circ} \mathrm{C}, 30 \%\right.$ soil field capacity); heat (T3: $32^{\circ} \mathrm{C}$, $80 \%$ soil field capacity); combined stress $\left(\mathrm{T} 4: 32^{\circ} \mathrm{C} 30 \%\right.$ soil

field capacity). The exudates are collected to the following root type-zone: seminal-apical ( $\mathbf{\square}),=$, seminal-subapical ( $\mathbf{})$,

primary-apical ( $\square$ ), primary-subapical ( $)$ ). The values are

reported as means \pm standard errors. Three way ANOVA with Tukey post hoc test: ${ }^{*} p=0.01-0.05,{ }^{* *} p=0.01-0.001$;

$* * * p<0.001$. 

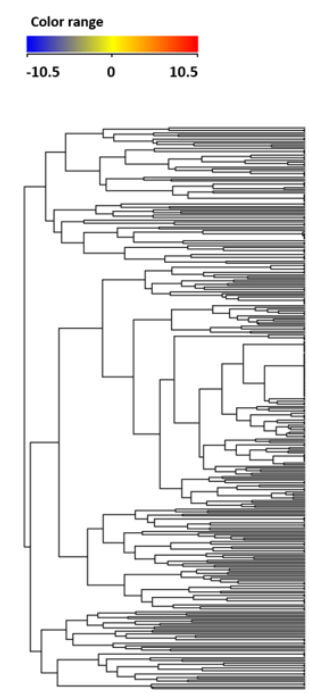

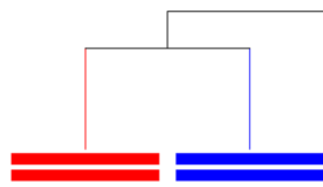

$\mathrm{T} 2$
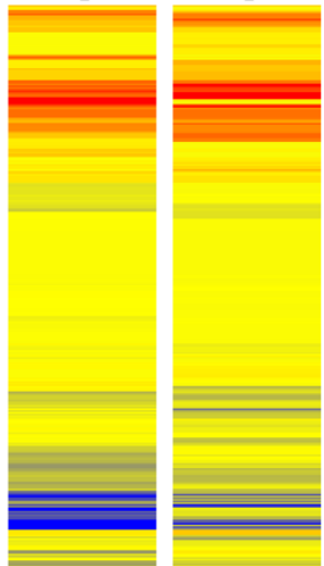

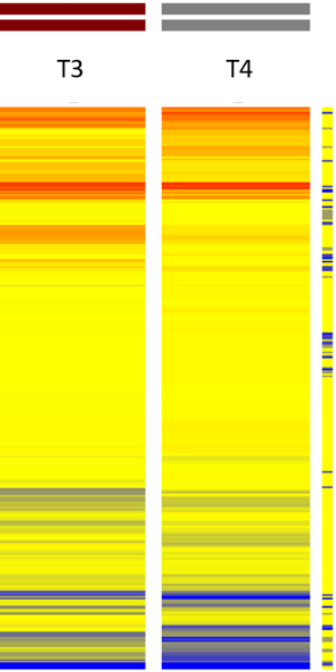

Figure 3. Unsupervised hierarchical cluster analysis (Euclidean distance; linkage rule: Ward) carried out from root exudates chemical profiles. Metabolites were obtained by UHPLC-ESI/QTOF-MS untargeted analysis, and their intensities used to build up the fold-change heatmap here provided.

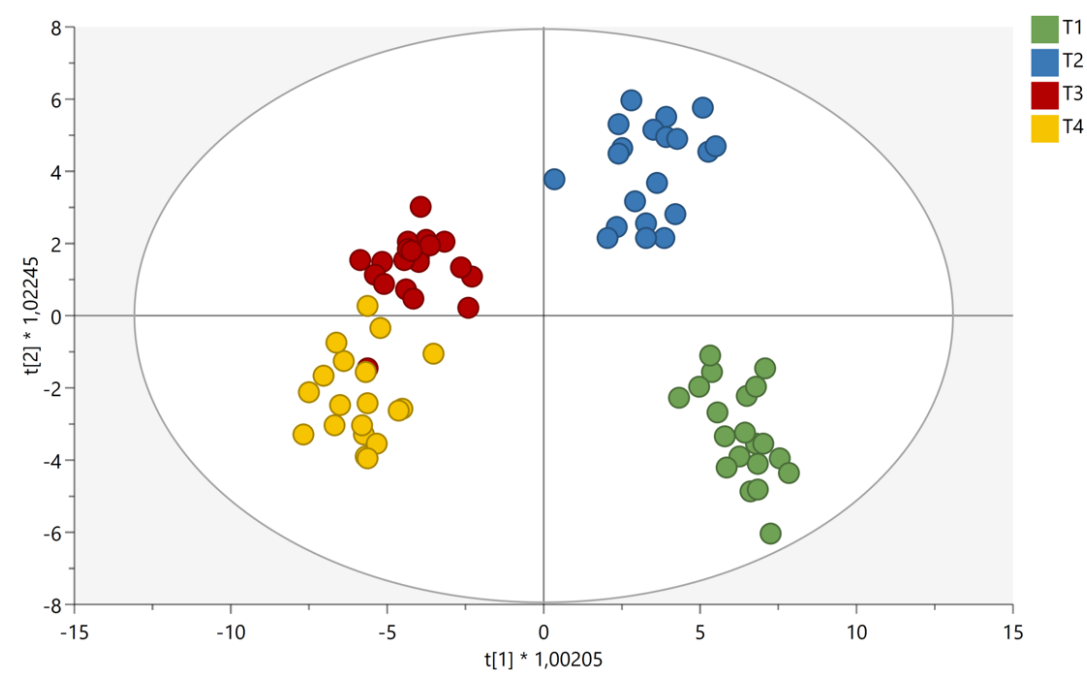

Figure 4. Score plot of Orthogonal projection to latent structures discriminant analysis (OPLSDA) supervised modelling carried out from untargeted metabolomics profiles of root exudates (correlation $R^{2} Y=0.83$ ), prediction ability $Q^{2} Y=0.48$ ). 
(a)

Up-exuded

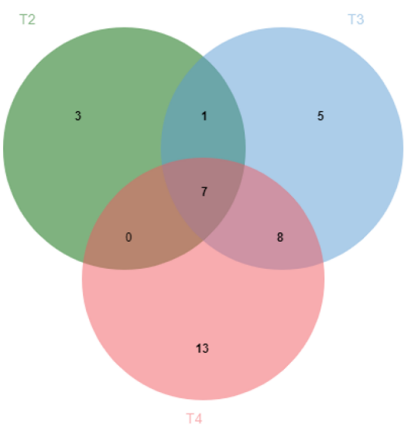

(b)

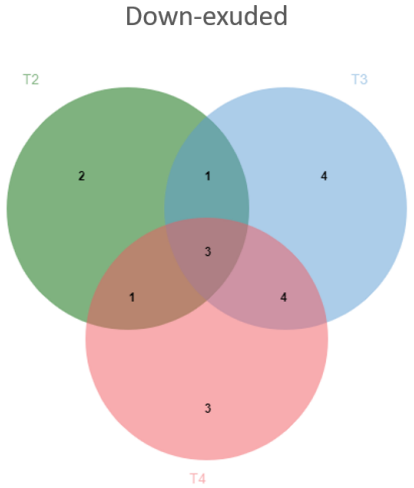

Figure 5. Venn diagram summarizing the metabolites differentially up-exuded (a) and down-exuded (b) that resulted from the volcano analysis. 